\title{
Non-Hodgkin lymphoma in celiac disease: causality or casuality on the scene?
}

\author{
Raffaella Mormile $^{1}$
}

Accepted: 30 September 2015 / Published online: 7 October 2015

(C) Springer-Verlag Berlin Heidelberg 2015

\section{Dear Editor:}

Celiac disease (CD) has been connected with a higher-thannormal risk of developing non-Hodgkin lymphoma (NHL) suggesting shared pathways of genetic predisposition to these pathologically distinct entities. However, understanding regarding molecular mechanisms underlying such association still remains unclear. CD is a chronic, immune-mediated condition of the small intestine caused by intolerance to dietary gluten from wheat and similar proteins in rye and barley that develops in genetically susceptible individuals. In $\mathrm{CD}$, a range of pro-inflammatory cytokines are secreted interferon- $\gamma$ (IFN- $-\gamma$ ) being a key determinant in gut permeability as well as in inflammation. It has been highlighted that IFN-y gene polymorphism may have a role in susceptibility to $\mathrm{CD}$. IFN-y secreted by gluten-activated celiac patient $\mathrm{T}$ cells has been described to be the primary effector of increased peptide translocation across the gut epithelium during active disease. In this contest, IFN- $\gamma$ is considered the most potent inducer of the endogenous extracellular enzyme, transglutaminase 2 (TG2). A number of lines of evidence advise that TG2 is a critical player in the pathogenesis of $\mathrm{CD}$ because it is able to deamidate gluten peptides at specific glutamine residues triggering the strong autoimmune response in the celiac intestine. IFN- $\gamma$ mediated activation of TG2 has been reported to require phospatidylinositol-3-kinase (PI3K) pathway activity. PI3K pathway has been shown to play key regulatory roles in many cellular processes such as cell survival, proliferation, and differ-

Raffaella Mormile

raffaellamormile@alice.it

1 Division of Pediatrics and Neonatology, Moscati Hospital, Via A. Gramsci, 3, 81031 Aversa, Italy entiation. Overexpression of TG2 has been shown to contribute not only to $\mathrm{CD}$ but also to a profile of cancers by constitutive activation of nuclear factor-kB (NF-kB). It is known that the $\mathrm{NF}-\mathrm{kB}$ route is constitutively upregulated in $\mathrm{CD}$. The NF-kB pathway has long been recognized to be a prototypical proinflammatory signalling pathway. Intriguingly, it has been detected that NF-kB pathway has the ability to cross-talk synergistically with PI3K pathway. NHL is a lymphoid malignant neoplasm with multiple subtypes. Patients typically suffer from persistent painless lymphadenopathy, but some individuals may present with constitutional symptoms or with involvement of organs other than the lymphoid and hematopoietic system. Although NHL is generally responsive to current therapeutic approaches, many subtypes remain treatable but are not curable. Accumulating data have emphasized the important role of PI3K in NHL, particularly in the disease initiation, progression, and survival. Furthermore, aberrant deregulated NF-kB activation is described as a hallmark of several lymphoid malignancies including NHL and is directly associated with advanced disease. Taken together into account, we speculate that common genetic variations in genes involved in IFN-y signalling pathways may modulate NHL risk in CD patients. We hypothesize that IFN-y allelic variants may play a causative role in lymphomagenesis by deregulating the functional network of PI3K and NF-kB signalling pathways. Therefore, IFN- $\gamma$ gene polymorphisms should be assessed in CD. The use of IFN- $\gamma$ gene polymorphism as a biochemical marker of neoplastic transformation might represent an important opportunity to precociously identify $\mathrm{CD}$ patients who are potentially at elevated risk for NHL development. Genomic and proteomic studies are required in order to define the components of the potential machinery leading to both the diseases by variants of IFN-gamma gene signalling. The strength for the future may be to implement strategies for PI3K and NF-kB upstream inhibition targeting pathways which are selectively relevant to prevent both CD and NHL. 